\title{
80 Anforderungen an die Laufschuhe
}

\author{
(c) Springer-Verlag GmbH Deutschland, ein Teil von Springer Nature 2018 \\ D. Mathias, Fit und gesund von 1 bis Hundert \\ https://doi.org/10.1007/978-3-662-56307-6_80
}

Die enormen Belastungen von Gelenken, Muskeln und Bindegeweben bei der Fortbewegung zeigen auf, welche große Bedeutung dem Schuhwerk zukommt. Ein falscher Schuh kann schnell Beschwerden verursachen, ebenso aber kann bei laufbedingten Schmerzen ein Wechsel der Laufschuhe Wunder bewirken. Leider gibt es nicht den Einheitsschuh für jeden Läufertypen. Gewicht, eventuelle Fußund Beinfehlstellungen oder falsche Fußführung sind Beispiele, warum immer die individuelle Auswahl getroffen werden muss. Grundsätzlich sollte ein Laufschuh den Fuß in seinen natürlichen Bewegungsabläufen so wenig wie möglich behindern. Eine flexible Sohle gewährleistet ein optimales Abrollen des Schuhs und damit eine gute Kraftübertragung vom Fuß auf den Boden. Steife Sohlen hebeln dagegen den Fuß und können zu Überlastungsschäden führen, weil vermehrt Arbeit auf die Zehen übertragen wird.

Sohlen und Absätze von Laufschuhen dürfen nicht zu dick und zu breit sein, weil sie dann Bodenunebenheiten verstärken und die Gefahr des Umknickens erhöhen. Dicke Sohlen belasten auch stark die Achillessehne, weil bei der seitwärts gerichteten Abrollbewegung größere Hebelkräfte wirksam werden. Leichte Schuhe können bei untrainierten Personen die Muskulatur zu stärkerer Haltearbeit zwingen. Für Anfänger sind wegen der besseren Fußführung festere Schuhe geeigneter.

Da sich der Laufschuh bei der Abrollbewegung verkürzt, darf er nicht zu klein gekauft werden. Der Spielraum für die Zehen sollte etwa $10 \mathrm{~mm}$ betragen.
Fehlt dieser Spielraum, dann können nach langen Anstrengungen blutunterlaufene, schmerzende Zehennägel und damit verbunden ein verkrampfter Laufstil die Folge sein. Der Laufschuh sollte eine individuell anpassbare Schnürung ermöglichen. $\mathrm{Zu}$ lockere Schnürungen sind häufig Ursache von Knie- und Schienbeinschmerzen, da der Fuß nicht fest in der Ferse gefasst wird. Andererseits können sehr schmerzhafte Druckbeschwerden auftreten, wenn die Schnürung zu fest auf dem hohen Fußrücken eines Hohlfußes aufsitzt. Für Läufer mit großem Trainingspensum ist es sinnvoll, abwechselnd mit verschiedenen Schuhen zu laufen. Gelenke und Muskeln werden so unterschiedlich belastet und dadurch teilweise geschont.

Die Verwendung verordneter Einlegesohlen zur akuten Therapie von Überlastungsschäden sollte in Abständen von mehreren Wochen bis einigen Monaten überprüft werden, da solche Sohlen durch Veränderungen der Biomechanik am Fuß ihrerseits Überlastungsschäden bewirken können. Ferner muss bedacht werden, dass die unteren Extremitäten auch im normalen Alltag erheblich belastet werden. Deshalb können die ständig getragenen Straßenschuhe ebenfalls Auslöser von Überlastungsschäden sein. Dies entgeht aber meist der Aufmerksamkeit der Betroffenen, weil sich die Schmerzen in der Regel zuerst bei den größeren Krafteinwirkungen während des Sports bemerkbar machen und in diesen Fällen dann fälschlicherweise die Laufschuhe angeschuldigt werden. Ein in diesem Zusammenhang besonderes Problem sind Stöckelschuhe. Frauen, die solche Schuhe tragen, leiden z. B. häufiger an muskulären Schmerzen und an Großzehenfehlstellungen (Hallux valgus). 